Methods 60 workers of a steel plant, aged 25-55 years, were enrolled in a panel study with two blood samples at the beginning and the end of a week. Gene expression was quantified by a 44-gene PCR Array and normalised by GAPDH housekeeping. Gene expression and PM10 data were log-transformed. Univariate correlation between each gene expression and PM10 is misleading because does not account for the association structure among genes. We instead focused on the entire association network among genes and the impact of PM10 exposure on it. Since our study is mainly explorative we deliberately choose a simple approach: the minimal BIC forest. This approach, which relies on Linearity, Multivariate Normality and the Adequacy of Linear Scores, is useful as a preliminary step towards understanding the overall dependence structure of high-dimensional discrete and/or continuous data.

Results The minimal BIC forest resulted in the identification of eight subgroups of correlated genes expression. The model including PM10 showed a negative association between PM10 exposure and NFKB1 expression. The sensitivity analysis on the assumptions was conducted as follows:

a. all possible [n=1892] Student t-statistics for squared terms were calculated;

b. all possible $[n=39732]$ choices of cross-product terms in the linear regressions.

No violation regarding the correlation between PM10 and gene expressions was found.

Conclusions The study was explorative and no a priori knowledge on gene expression pathways was considered. In conclusion we found an association of PM10 exposure with NFKB1 expression in a occupational context of high ambient concentrations.

\section{MYTH OR REALITY! ACUTE LEAD POISONING - A CASE REPORT}

M Lghabi*, W Allouche, A Ichane, B Benali, A El Kholti. Faculty of Medicine and Pharmacy of Casablanca, Casablanca, Morocco

\subsection{6/oemed-2018-ICOHabstracts.1142}

Introduction Lead is a metal used since ancient times in many applications. Lead poisoning can be acute resulting from brief exposure to very high amounts of lead or chronic, due to prolonged exposure to small amounts.

Observation We report a case concerning a 27-year-old man who had been working in the car battery crushing for 8 months in a factory (informal sector) with no pathological history and lead poisoning. It's about a 27-year-old man who has been working in the crushing of car batteries for 8 months in a factory (informal sector), without a particular pathological history, which has lead poisoning. He was a victim of acute lead poisoning that was clinically manifested by a sub-occlusive syndrome associated with anaemic syndrome evolving in a context of apyrexia. Faced with the suspicion of lead poisoning, the patient was referred to the Occupational Health Service where the lead origin was confirmed (Lead, ALAU and PPZ elevated). The development was favourable after a chelating treatment provided by the Moroccan anti poison centre (MAPC).

Discussion Acute lead poisoning in the workplace is rare but still exists, manifested in particular by digestive disorders, neurological effects which are serious and anaemia. In Morocco, there are no statistical data on the level of lead impregnation. In our case, a fact-finding mission was organised by the MAPC, following which it was recommended that the plant should be quickly shut down due to the seriousness of the findings and regulatory and administrative non-conformities. His disease was declared as a disease of occupational origin in accordance with the Moroccan legislation in force (table 1.1.1 of occupational diseases).

Conclusion Lead poisoning is a frequently overlooked public health problem, and collaboration between the attending physician and the occupational physician can help identify, detect early and prevent risky situations.

\section{COACHBUILDING AND PAINTING SHOP WORKERS' CO- EXPOSITIONTO NOISE AND OTOTOXIC SUBSTANCES. RISK EVALUATION AND PREVENTION}

${ }^{1}$ Renée Daumas*, ${ }^{2}$ Thomas Venet. ${ }^{1}$ CMTI Santé Travail 06, Nice, France; ${ }^{2}$ INRS (Institut National de Recherche et de sécurité), Vandœuvre, France

\subsection{6/oemed-2018-ICOHabstracts.1143}

Introduction Coachbuilding and painting shop workers are coexposed to noise and ototoxic substances thereby producing synergistic adverse effects on hearing, which might increase the risk of professional hearing loss. A previous investigation showed that most of employers and workers ignore those risks due to occupational activities, or negligences.

Methods 60 volunteers were measured in 24 shops. We used dosimeters for evaluating the noise exposures, and passive badges for the amount of solvents inhaled by each worker during a workday.

CAS DPOAE suppression (Echoscan) allowed auditory fatigue to be assessed by summing several sources: metabolic fatigue from hair cells, afferent and efferent fibres, central nuclei (SOC and facial), and obviously from middle-ear muscles. The trigger threshold variations of workers exposed to both noise and solvents were measured with Echoscan twice a day.

In the meantime, the wearing of auditory and respiratory individual protectors was monitored.

Results The Lex $8 \mathrm{~h}$ measurements were higher than 80, 85, $87 \mathrm{~dB}(\mathrm{~A})$ for $33,17,12 \%$ of tested workers, respectively.

Toluene, ethylbenzene and xylene constituted the most used ototoxics. These findings are in agreement with the data collected in the French database COLCHIC for carshops' workers. Taken separately each solvent exposure was inferior to its legal threshold limit value, but an exposure to a chemical cocktail may affect the hair cells or the central nuclei. Results showed that data obtained with Echoscan were sensitive both to noise and solvents. At the end of a working day, trigger threshold variations of workers exposed to both noise and solvents were significantly lower than those measured in workers exposed to noise only.

Workers and shop observation showed significant neglects in collective and individual prevention.

Conclusion Difficulties to characterise workers' effective exposure are numerous in car shops. Regarding employers and workers' awareness, a primary prevention has been launched through information sessions. Auditory fatigue depends on the nature of the exposure. A co-exposure impacts the trigger threshold variations of CAS DPOAEs. 